\title{
Exciton ionization in a quantum well studied by surface acoustic waves
}

\author{
C. Rocke, A. O. Govorov, ${ }^{*}$ and A. Wixforth \\ Sektion Physik der LMU, Geschwister-Scholl-Platz 1, D-80539 München, Germany \\ G. Böhm and G. Weimann ${ }^{\dagger}$ \\ Walter-Schottky-Institut der TUM, D-85748 Garching, Germany
}

(Received 22 December 1997)

\begin{abstract}
Photogenerated excitons in a semiconductor quantum well are ionized by the strong lateral piezoelectric field of an intense surface acoustic wave. The ionization process is directly observed by the absorption of a second sound wave probing the areal conductivity of the photogenerated charges. Above a certain threshold field strength of the pumping surface wave the neutral excitons are dissociated into electron-hole pairs, which is indicated by a sudden onset of absorption of the probe wave. By selectively tuning the energy of the exciting laser, the dissociation of both quasi two-dimensional excitons in the quantum well as well as three-dimensional excitons in the barrier material can be observed. [S0163-1829(98)52412-5]
\end{abstract}

The electric-field dependence of optical absorption near the band edge of semiconductors has been extensively studied during the last decade. Great interest in optical computing and the need for fast telecommunication techniques have triggered the development of various devices for modulation, detection, and all-optical switching. ${ }^{1}$ Many of the above devices, based on layered, band-gap-engineered semiconductor structures make use of the fact that strong electric fields modify their optical properties. For example, an electric field applied perpendicular to a quantum well (QW) results in the so-called quantum confined Stark effect (QCSE). ${ }^{2}$ On the other hand, lateral electric fields imposed by laterally patterned gate electrodes inducing a lateral potential superlattice have been shown to result in the two-dimensional FranzKeldysh effect and can lead to field ionization of the photogenerated excitons in the quantum well. ${ }^{3}$ More recently, we demonstrated also that surface acoustic waves (SAW) on a piezoelectric semiconductor substrate can act as such a lateral superlattice and effectively ionizes the photogenerated excitons. ${ }^{4,5}$ The acoustically induced moving potential grating is able to transport the fragments of the exciton ionization process, free electron-hole pairs, over macroscopic distances. ${ }^{5}$ At the same time, the study of SAW absorption is a very sensitive method to directly observe the presence of free electrons and holes. ${ }^{6}$ Here, we combine these two effects, the SAW-induced ionization of excitons and the SAWabsorption by free carriers.

The aim of this paper is to report on the direct observation of the dissociation process of the photogenerated excitons in a semiconductor quantum well under the influence of an intense piezoactive surface sound wave. For this purpose, the sheet conductivity of the photogenerated charges is probed by the absorption of a second acoustic wave transversing the excitation region on the sample. As has been pointed out before, SAW absorption measurements are particularly sensitive at very low conductivities, a fact that has been used before to study the dynamical conductivity of quasi-twodimensional electron systems (Q2DES) in the regime of the quantum Hall effect. ${ }^{6-8}$

The design of our sample is shown in Fig. 1. SAWs are excited by means of interdigital transducers (IDTs) on the (100) surface of our semiconductor quantum well system, propagating along the [110] direction. The undoped quantum well samples used in our experiments are grown by molecular beam epitaxy on a semi-insulating GaAs substrate. The well consists of $10 \mathrm{~nm}$ strained $\mathrm{In}_{0.15} \mathrm{Ga}_{0.85}$ As grown on a 1 $\mu \mathrm{m}$ thick GaAs buffer and is covered by a $20 \mathrm{~nm}$ thick GaAs cap layer. The active area of the sample is etched into a cross-shaped $2.5 \mathrm{~mm}$ long and $0.3 \mathrm{~mm}$ wide mesa with two IDTs each at the ends of the cross arms. Two facing IDTs are designed to operate at a center frequency $f_{S A W, 1}=420 \mathrm{MHz}$ $\left(\lambda_{S A W 1}=6.8 \mu \mathrm{m}\right)$, the other two at $f_{S A W, 2}=840 \mathrm{MHz}$ $\left(\lambda_{S A W 2}=3.4 \mu \mathrm{m}\right)$. They are partially impedance matched to the $50 \Omega$ radio frequency (RF) circuitry using an on-chip matching network thus strongly reducing the insertion loss of each transducer. Our sample is mounted in an optical cryostat and the experiments presented here are performed at $T=5 \mathrm{~K}$. Application of a high frequency signal to one of the IDTs launches a SAW that can be detected at the other IDT after an acoustic delay of the order of $1 \mu$ s determined by the spacing of the IDTs. Either pulsed or continuous wave (cw)

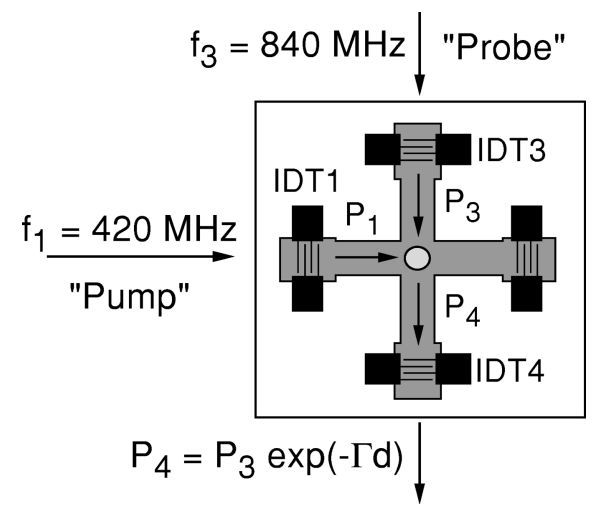

FIG. 1. Schematic illustration of the design of the sample used in the described experiments. Surface acoustic waves are excited on the In-Ga-As/GaAs QW sample using interdigital transducers and intercept at the center of the cross-shaped mesa, where optical excitation is performed. 


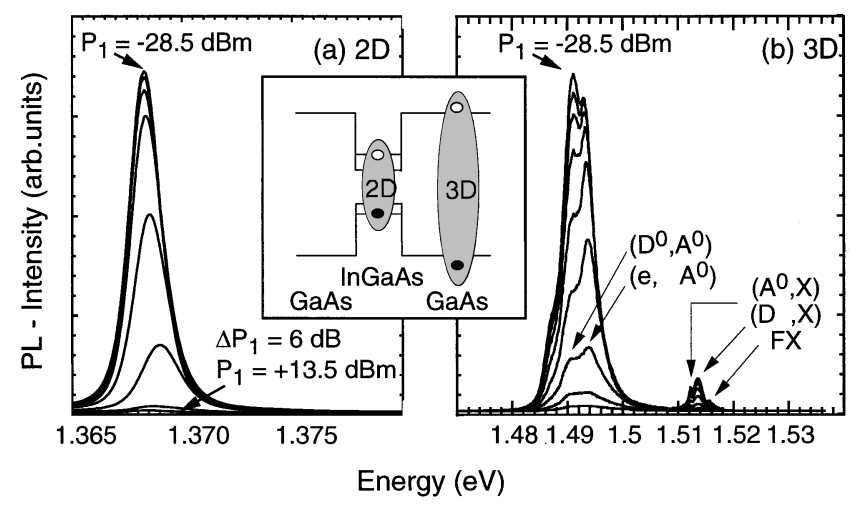

FIG. 2. Time-integrated PL-spectra of excitonic interband transitions of the In-Ga-As QW (a) and in the barrier (bulk) GaAs material (b). As the SAW power $P_{1}$ is increased above a critical threshold power, the PL intensities strongy decrease due to the efficient dissociation in the acoustically induced lateral electric field. An offset between traces (a) and (b) is introduced for clarity.

operation of the SAW transducers is possible. Both SAWs intercept at the center of the cross-shaped mesa structure. Light from a titanium-sapphire laser is used for optical interband excitation in an area of $A=\pi(100 \mu \mathrm{m})^{2}$ in the center of the cross-shaped mesa. The photoluminescence (PL) of the sample is analyzed in a triple grating spectrometer. Either a photomultiplier or a charged coupled device (CCD) serve as a detector.

As has been demonstrated before, the lateral piezoelectric fields of a SAW can be strong enough to field ionize photogenerated excitons in a quantum well. ${ }^{4,5}$ The resulting free electrons and free holes are then spatially separated and efficiently trapped in the wells of their respective potential minima induced by the SAW. Thus, the recombination probability of the electrons and holes is greatly suppressed, resulting in a quenched PL intensity. This ionization and subsequent quenching of the PL under the influence of a SAW is shown in Fig. 2. Both for quasi-two-dimensional quantum well excitons (a), as well as for three-dimensional excitons in the bulk (b), a strong reduction of the PL intensity is observed, if the SAW power is increased. For 3D excitons, this reduction has also been observed recently by another group, ${ }^{9}$ where the various quenching behavior of bound and free excitons has been discussed in detail. Our experimental results quantitatively agree with those obtained there. For the highest pump power levels shown in the figure, a complete suppression of the PL both in the quantum well and in the bulk can be achieved.

The inset of Fig. 3 shows the observed PL intensity for an optical excitation energy $E_{\text {las }}=1.59 \mathrm{eV}$ and $P_{\text {las }}=5 \mu \mathrm{W}$ under the influence of the "pumping" SAW. We plot the intensity of different PL lines versus the power of the incident SAW in the regime between $P_{1}=-20 \mathrm{dBm}$ and $P_{1}=$ $+10 \mathrm{dBm}$. For lower power levels, that have been omitted in the inset, the PL remains nearly unaffected, whereas for $P_{1}$ higher than $-20 \mathrm{dBm}$ a strong decrease of the PL signals is observed. It is interesting to note that the ionization thresholds for the different excitonic lines differ by a considerable amount. In the case of bulk excitons, this observation is consistent with the one reported in Ref. 9, where a complete quenching of the PL, however, has not been observed.

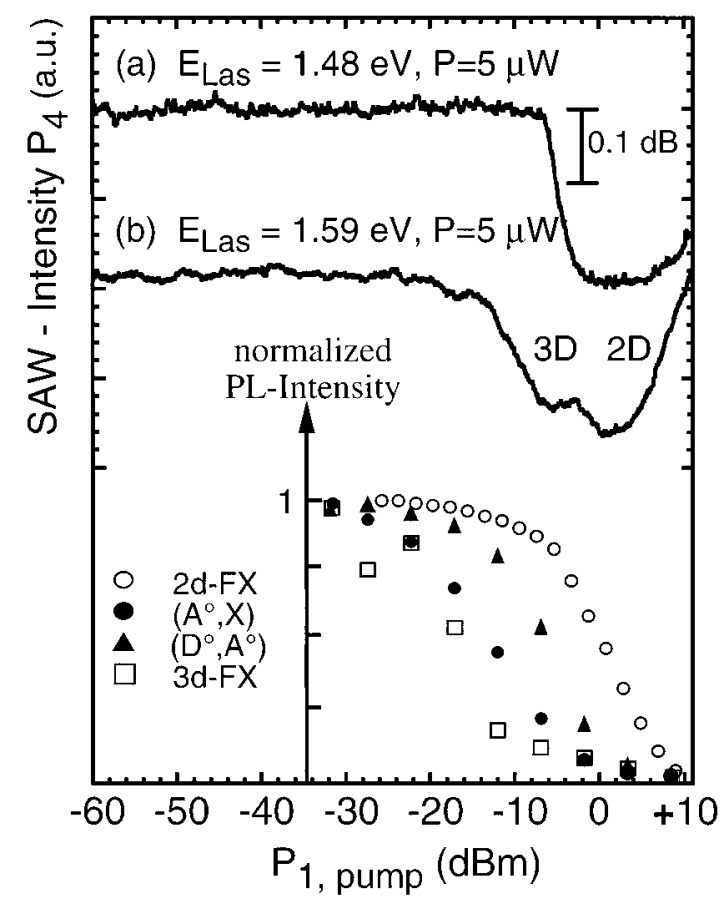

FIG. 3. Transmitted SAW intensity $P_{4}$ of the "probe" SAW as a function of the incident pump SAW intensity $P_{1}$. Above a critical pump intensity, the optically generated excitons are effectively dissociated into separated stripes of electrons and holes, which results in a sudden onset of the attenuation of the probe pulse. The inset depicts the PL intensity variation of the different transitions in Fig. 2 with acoustic power $P_{1}$.

The fact that the ionization thresholds of PL intensity in the inset of Fig. 3 are different for different excitons can be understood in terms of the exciton binding energy $E_{B}$. We can expect that excitons with larger binding energy are more stable against dissociation by an electric field. While $E_{B}$ for free quasi-2D excitons in In-Ga-As QWs is about 8-9 $\mathrm{meV},{ }^{10}$ the corresponding value for free excitons in bulk GaAs is only about $5 \mathrm{meV}$. Thus the ionization threshold power for 2D-excitons is larger than the one for free bulk excitons. Similarly, the bound bulk exciton lines in the inset of Fig. 3 are suppressed at higher SAW powers in comparison with free bulk excitons because the binding to impurities also increases $E_{B}$.

We now focus on the observation of the exciton dissociation into free electrons and holes being trapped and confined in the traveling potential wells of the propagating intense SAW. For this purpose we monitor the transmitted intensity $P_{4}$ of a second SAW propagating perpendicularly to the pump SAW as a function of the acoustic power $P_{1}$ of the latter. On the length scale of the SAW, the optically generated excitons behave like neutral particles as their effective Bohr radius is much smaller than $\lambda_{S A W}$. The areal conductivity related to the field induced polarization of the excitons is negligible, so we do not expect any screening of the piezoelectric potential of the transmitted SAW and hence no attenuation as described in Ref. 6 However, once the excitons are field ionized, their "fragments"-electrons and holes--are spatially separated and subsequently confined in the lateral potential wells of the pump SAW. Now the probe SAW encounters many parallel stripes of free electrons and 
holes, resembling a lateral nipi structure with a period $\lambda_{S A W 1}$. Those electrons and holes are free to move along the direction normal to the propagation of the pumping and confining SAW and are therefore expected to interact with the second SAW according to their areal conductivity.

This is exactly what we observe experimentally. The result is shown in Fig. 3, where we plot the transmitted SAW intensity $P_{4}=P_{3} \exp (-\Gamma d)$ as a function of the power $P_{1}$ of the pumping SAW and for two different optical excitation energies $E_{\text {las }}$, as will be discussed below. $P_{3}$ is the incident power of the "probe" SAW, $d$ is the length of the interaction region, which in our case corresponds to the width of the pumping SAW path, namely $d=0.3 \mathrm{~mm}$. For a $2 \mathrm{D}$ system the attenuation coefficient $\Gamma$ is given by ${ }^{6}$

$$
\Gamma=k \frac{K_{e f f}^{2}}{2} \frac{\sigma / \sigma_{M}}{1+\left(\sigma / \sigma_{M}\right)^{2}} .
$$

Here, $k=2 \pi / \lambda_{S A W}$ denotes the SAW wave vector, $K_{e f f}^{2}$ $=6.4 \times 10^{-4}$ is the electromechanical coupling constant for GaAs and the given crystal orientation, $\sigma$ is the sheet conductivity of a two-dimensional conducting layer close to the crystal surface where the SAW is propagating, and $\sigma_{M}$ $=3.4 \times 10^{-7} \Omega^{-1}$, a critical conductivity where maximum attenuation occurs. We believe that for the experiment described here, expression (1) is a good approximation even for the case of the 3D excitons and of the lateral and moving 2D nipi structure, as the stripes of electrons and holes confined in the potential wells of the SAW are still way too wide to exhibit one-dimensional behavior. The relevant conductivity $\sigma$ in (1), however, should be taken as an averaged conductivity of both electrons and holes. Also for the 3D excitons, Eq. (1) may be qualitatively used to describe the SAW absorption as the thickness of the photogenerated charge layer is still smaller than the wavelength of the SAW.

At very low pumping power levels $P_{1}$, the probing SAW is nearly unaffected as indicated by a constant transmitted SAW intensity $P_{4}$ over several decades of $P_{1}$. However, for pumping power levels greater than approximately $P_{1}=$ $-10 \mathrm{dBm}$, a strong decrease of the transmitted SAW intensity $P_{4}$ or equivalently an increase of the attenuation coefficient $\Gamma$ is observed. This decrease of the probe SAW intensity is strongly dependent on the energy $E_{\text {las }}$ of the exciting laser, as can be seen in the upper two traces in Fig. 3.

In Fig. 3(a), the energy of the incident laser $E_{\text {las }}$ $=1.48 \mathrm{eV}$ is chosen such that only the In-Ga-As quantum well is optically excited, resulting in a selective population of the QW by photogenerated excitons. For low acoustic pump powers $P_{1}$, their quasistatic conductivity in the QW is negligible, indicated by a constant probe intensity $P_{4}$. However, for $P_{1}>-6 \mathrm{dBm}$, corresponding to a lateral piezoelectric field strength of $E_{1}>600 \mathrm{~V} / \mathrm{cm}$, the probability for the dissociation of the excitons by field-induced tunneling processes rapidly increases which is marked by a sharp onset of the absorption of the probe SAW. This change in the SAW transmission can be understood by a process as described by Eq. (1). The point of minimum transmission (or equivalently maximum attenuation) corresponds to the situation, where the averaged conductivity $\sigma$ of the spatially separated electrons and holes reaches $\sigma_{M}$ as defined above. Further increase of the pumping power $P_{1}$ results in an increase of the carrier densities and thus, caused by an increased screening, in a smaller interaction between the probe SAW and the mobile carriers. The result is a significantly reduced attenuation coefficient $\Gamma$.

Optical excitation at a laser energy of $E_{\text {las }}=1.59 \mathrm{eV}$ [see Fig. 3(b)] being larger than the band gap of the quantum well barriers leads to the generation of both $3 \mathrm{D}$ excitons in this GaAs barrier (bulk) material and in the In-Ga-As quantum well. This simultaneous generation of $2 \mathrm{D}$ and $3 \mathrm{D}$ excitons is clearly observed in our experiment, as the transmitted SAW intensity exhibits two well defined minima at about $P_{1}=$ $-8 \mathrm{dBm}$ and $P_{1}=0 \mathrm{dBm}$. Those minima correspond to maxima in the SAW attenuation $\Gamma$ as described above. The onset of the SAW attenuation, however, is now shifted towards lower pumping powers of $P_{1}=-13 \mathrm{dBm}$, corresponding to a piezoelectric field strength of only $E_{1}$ $=300 \mathrm{~V} / \mathrm{cm}$. This attenuation at smaller pumping powers can be attributed to the dissociation of the $3 \mathrm{D}$ excitons in the GaAs barrier material. As the exciton binding energy of 3D excitons is considerably smaller than the one for the $2 \mathrm{D}$ excitons in the In-Ga-As quantum well, the piezoelectric fields necessary for ionization will also be smaller. Caused by the variation of the SAW-induced fields with depth into the crystal, the dissociation rate of the $3 \mathrm{D}$ excitons also varies with depth. Both this field strength variation as well as the presence of many different excitonic states with different binding energies in 3D will result in a somewhat smoother onset of SAW attenuation in the $3 \mathrm{D}$ case as compared to the $2 \mathrm{D}$ case. Note, that the onset of exciton ionization, indicated by the onset in the SAW absorption, agrees very well with the decrease of the PL intensity of the most intense neutral acceptor bound exciton $\left(A^{0}, X\right)$ as shown in the inset of Fig. 3 .

As was mentioned before, the quenching of the PL arises from field ionization of excitons in the presence of an intensive SAW. It is interesting to estimate the tunneling ionization time of excitons for the characteristic SAW power when the SAW starts to quench the PL signal. For example, the 2D free exciton PL intensity drops to the half of its initial value (see inset of Fig. 3) at the SAW power of $P_{1}=-1 \mathrm{dBm}$. The corresponding amplitude of an in-plane electric field $F_{\|}$induced by SAW can be calculated and is about $1 \mathrm{kV} / \mathrm{cm}$. The tunneling ionization time for free $2 \mathrm{D}$ excitons $\tau_{\text {ion }}$ in a uniform electric field is given by ${ }^{2}$

$$
\tau_{i o n} \simeq \frac{\sqrt{\pi}}{64} \frac{\hbar}{R}\left(\frac{e F_{\|} a_{0}^{*}}{R}\right)^{1 / 2} \exp \left(\frac{4}{3} E_{B} \frac{\sqrt{2 \mu E_{B}}}{\hbar e F_{\|}}\right)
$$

where $a_{0}^{*}=\epsilon \hbar^{2} / e^{2} \mu=14 \mathrm{~nm}$ is the effective Bohr radius, $\mu^{-1}=m_{e}^{-1}+m_{h}^{-1}$ is the reduced mass, and $R=\mu e^{4} / 2 \hbar^{2} \epsilon^{2}$. Using $m_{e}=0.063 m_{0}, m_{h}=m_{h h}=0.2 m_{0}, \epsilon=13$, and $E_{B}$ $\approx 8.5 \mathrm{meV}^{10}$ we get from Eq. (2) an estimation $\tau_{\text {ion }}$ $\approx 0.35 \mathrm{~ns}$ for an in-plane electric field of $1 \mathrm{kV} / \mathrm{cm}$. We can suppose that the excitons can be effectively ionized in free electrons and holes if the exciton life-time due to radiative processes $\tau_{\text {rad }}$ at zero electric field is comparable or greater than the tunneling-ionization time. The measured value of $\tau_{\text {rad }}$ in time-resolved experiments on GaAs-based QWs at low temperatures is in the range of $0.4-1.5 \mathrm{~ns} .^{10-12}$ Thus one can see that our estimation for $\tau_{i o n}$ is of the same order of 
magnitude as $\tau_{\text {rad }}$. This is consistent with our understanding of SAW-induced effects in the optical spectra.

In summary, the ionization of photogenerated excitons in a semiconductor quantum well sample by the strong lateral piezoelectric field of an intense surface acoustic wave has been investigated. Using a SAW pump and probe technique, the ionization process could be directly and sensitively monitored by a conductivity change during the transition of a neutral exciton gas into a spatially separated electron and hole plasma. Excitation at different laser energies allowed for a selective generation of excitons in the quantum well and the barrier material. Both 3D GaAs bulk excitons and quasi-2D InGaAs quantum well excitons and their field ionizations could be observed. The lateral ionization threshold field for the 3D excitons turned out to be smaller by about a factor of two as compared to the 2D case, in agreement with their different binding energies.

We gratefully acknowledge fruitful discussions with J. P. Kotthaus, technical advice of S. Manus and generous financial support by the Deutsche Forschungsgemeinschaft and the Bayerische Forschungsstiftung FOROPTO.
*Permanent address: Institute of Semiconductor Physics, 630090 Novosibirsk, Russia.

†Permanent address: Fraunhofer Institut IAF, D-79108 Freiburg, Germany.

${ }^{1}$ S. Schmitt-Rink, D. S. Chemla, and D. A. B. Miller, Adv. Phys. 38, 89 (1989).

${ }^{2}$ D. A. B. Miller, D. S. Chemla, T. C. Damen, A. C. Gossard, W. Wiegmann, T. H. Wood, and C. A. Burrus, Phys. Rev. B 32, 1043 (1985).

${ }^{3}$ A. Schmeller, W. Hansen, J. P. Kotthaus, G. Tränkle, and G. Weimann, Appl. Phys. Lett. 64, 330 (1994).

${ }^{4}$ C. Rocke, A. Wixforth, J. P. Kotthaus, W. Klein, G. Bohm, and G. Weimann, in Compound Semiconductors 1996, edited by M. S. Shur and E. A. Suris, IOP Conf. Ser. No. 155 (IOP Publishing, Bristol, 1997), p. 125.

${ }^{5}$ C. Rocke, S. Zimmermann, A. Wixforth, J. P. Kotthaus, G.
Bohm, and G. Weimann, Phys. Rev. Lett. 78, 4099 (1997).

${ }^{6}$ A. Wixforth, J. Scriba, M. Wassermeier, J. P. Kotthaus, G. Weimann, and W. Schlapp, Phys. Rev. B 40, 7874 (1989).

${ }^{7}$ V. W. Rampton, K. McEnaney, A. G. Kozorezov, P. J. A. Carter, C. D. W. Wilkinson, M. Henini, and O. H. Hughes, Semicond. Sci. Technol. 7, 641 (1992).

${ }^{8}$ R. L. Willett, Semicond. Sci. Technol. 12, 495 (1997).

${ }^{9}$ K. S. Zhuravlev, D. V. Petrov, Yu. B. Bolkhovityanov, and N. S. Rudaja, Appl. Phys. Lett. 70, 3389 (1997).

${ }^{10}$ D. Orani, A. Polimehi, A. Patane, M. Capizzi, F. Martelli, A. D'Andrea, N. Tomassini, P. Borri, M. Gurioli, and M. Colocci, Phys. Status Solidi A (to be published).

${ }^{11}$ J. Feldmann, G. Peter, E. O. Goebel, P. Dawson, K. Moore, C. Foxon, and R. J. Elliott, Phys. Rev. Lett. 59, 2337 (1987).

${ }^{12}$ I. Aksenov, Y. Aoyagi, J. Kusano, T. Sugano, T. Yasuda, and Y. Segawa, Phys. Rev. B 52, 17430 (1995). 